Fundamentos para el desarrollo profesional de los profesores de inglés. 2å. Edición. Pasto: Editorial Universitaria - Universidad de Nariño. (361 pp.). 2015. ISBN 978-958-8609-98-0.

APA citation style: Benavides B., Jorge E. (2015). Las Pruebas Estandarizadas como Forma de Medición del Nivel de Inglés en la Educación Colombiana. En J. Bastidas \& G. Muñoz (Eds.), Fundamentos para el desarrollo profesional de los profesores de inglés (pp. 2956). 2a. Edición. Pasto: Editorial Universitaria - Universidad de Nariño.

\title{
Las Pruebas Estandarizadas Como Forma de Medición del Nivel de Inglés en la Educación Colombiana
}

\author{
Jorge E. Benavides Burgos (joelbebu@gmail.com) \\ Departamento de Lingüística e Idiomas \\ Universidad de Nariño, San Juan de Pasto, Colombia
}

\section{Resumen}

El presente artículo trata sobre la importancia de las pruebas estandarizadas como medio de evaluación y medición del aprendizaje y nivel de conocimiento y uso de las lenguas extranjeras, específicamente el inglés. Se sabe que desde la segunda mitad del siglo XX los mecanismos para determinar con un buen nivel de validez y confiabilidad la competencia lingüística y comunicativa de una persona fueron pruebas que iniciaron un proceso de investigación de campo y de revisión continua para asegurar su calidad. Hoy en un mundo globalizado y con muchas posibilidades para estudiantes, profesores, e investigadores se han popularizado e institucionalizado este tipo de pruebas en los diferentes países del mundo. Colombia no ha sido la excepción puesto que desde comienzos de la década del 2000 el Ministerio de Educación Nacional se ha acogido al estándar promovido por el Marco Común Europeo de referencia para las Lenguas, que permite estandarizar los niveles de medición de aprendizaje de la lengua extranjera en estudiantes, profesiones y profesionales en general.

\section{Abstract}

This article discusses the importance of standardized testing as a means of assessing learning and determining the level of knowledge and use of foreign languages like English. It is very well known that since the second half of the twentieth century efforts were made in order to come up with 
Fundamentos para el desarrollo profesional de los profesores de inglés. 2a adición. Pasto: Editorial Universitaria - Universidad de Nariño. (361 pp.). 2015. ISBN 978-958-8609-98-0.

valid and reliable measuring instruments for linguistic and communicative competence of a person in foreign languages. This began in the area of field research and ongoing reviewing processes to ensure the quality of testing procedures. Today, in a globalized world with many possibilities for mobility of students, teachers, and researchers standardized tests have been popularized and institutionalized in different countries. Colombia has not been the exception and starting from the beginning of this century the Ministry of Education has implemented the standard promoted by the Common European Framework of Reference for Languages which enables standardized measuring levels of English as a foreign language in students, teachers and professionals in general.

\section{Introducción}

Se ha especulado mucho sobre la incidencia de las pruebas estandarizadas dentro del proceso de aprendizaje de una lengua como medio para el control de calidad y eficacia de un determinado sistema educativo, especialmente en el área de la educación de las lenguas extranjeras en el mundo moderno.

Se conoce también que todo proceso programático en educación requiere de una evaluación (relacionada también con el concepto de medición para fines de este artículo) para comprobar que los objetivos del mismo se cumplan ya sea a corto, mediano o largo plazo. Esta evaluación debe estar apoyada por criterios válidos y confiables de medición, puesto que básicamente se evalúan los objetivos del proceso y las metas de un programa. El proceso está conformado por una serie de etapas o niveles en los que se supone se cumplen ciertos objetivos generales y específicos. Esto lamentablemente ha sido descuidado sistemáticamente en nuestro sistema educativo en lo que atañe a la lengua puesto que se desconoce en cada nivel el grado de aprendizaje (competencia lingüística y comunicativa) de las diferentes habilidades y aspectos lingüísticos como la 
Fundamentos para el desarrollo profesional de los profesores de inglés. 2a adición. Pasto: Editorial Universitaria - Universidad de Nariño. (361 pp.). 2015. ISBN 978-958-8609-98-0.

comprensión de lectura, la comprensión de escucha, la producción oral, la composición escrita, la gramática, el vocabulario, etc. de los participantes.

Se conoce también que la relación entre objetivos y criterios de evaluación no comporta congruencia y por consiguiente la confiabilidad tanto del cumplimiento de los objetivos como de la evaluación son cuestionables. A esto se suma la complejidad de evaluación de la lengua especialmente en aspectos de competencia y actuación lingüísticas donde la intersección de diferentes áreas, aspectos y categorías hacen de esta tarea algo más que un reto.

Las pruebas que informalmente se realizan como evaluación diaria o por periodos cortos no tienen la sistematicidad con la que se podrían determinar objetivos, y criterios de logro en cada una de las habilidades de la lengua. La parte secuencial en el proceso de la evaluación en cada una de las etapas del proceso de aprendizaje ya sea en períodos bimestrales, trimestrales o semestrales y ciclos largos como en periodos anuales también se ha descuidado constantemente.

\section{La necesidad de evaluar}

Es suficientemente conocido que la etapa de evaluación que implica medición del aprendizaje tiene un alto grado de importancia dentro de todo sistema o proceso de enseñanza aprendizaje puesto que se trata del proceso por medio del que se verifica el producto o resultado tanto de los objetivos como de la estructura de un curso o plan programático determinado. De esta manera cobra vigencia la conocida frase que dice, "Enseñar sin evaluar es como caminar sin avanzar."

Tanto el concepto o teoría de la evaluación y la práctica de evaluar como medios hacia un fin hacen parte de la historia del hombre que necesita saber o conocer el resultado o producto de un proceso de enseñanza dado, sobre todo si este implica un aprendizaje y una formación del individuo. Desde este punto de vista el hombre siempre ha deseado mejorar sus técnicas de medición tendientes a tomar las mejores 
Fundamentos para el desarrollo profesional de los profesores de inglés. 2aa. Edición. Pasto: Editorial Universitaria - Universidad de Nariño. (361 pp.). 2015. ISBN 978-958-8609-98-0.

decisiones de mejoramiento tanto a nivel de enseñanza como de aprendizaje. Estas decisiones son esenciales puesto que la historia nos dice que un gran porcentaje del comportamiento humano se habría basado en información errónea (Storey, 1970). Esta clase de información hace que el hombre busque afanosamente información válida sobre la cual tomar las mejores decisiones para un cambio pertinente. Sin embargo, la información válida es difícil de obtener, como lo manifiesta Thorndike (1997) debido al gran número de variables y situaciones que pueden ocurrir en un determinado contexto educativo.

\section{Las pruebas estandarizadas como evaluación sistemática}

La evaluación o medición, como se la conoce hoy, inició prácticamente con los conceptos de la medición en las ciencias físicas o naturales (Hays, 1973) y claramente una de las características de las ciencias naturales o físicas son sus métodos de medición, que consecuentemente se aplicaron a las ciencias sociales debido al éxito obtenido en el primer caso. El desarrollo y aplicación de técnicas de medición confiables y válidas en la educación durante los últimos años ha sido por demás inadecuado tanto por parte de instructores como de administradores educativos, algo que ha dejado a los profesores en un ámbito de desconocimiento de los procesos necesarios para afrontar la medición del aprendizaje en el aula de clase y por ende de los sistemas que lo integran (Tuckman, 1999).

Con respecto a las pruebas estandarizadas en general, como lo manifiesta Storey se han tenido dos etapas importantes en la historia de la medición del comportamiento humano en las ciencias sociales como en psicología y luego en sociología y subsecuentemente en todas las ciencias del comportamiento. En primer lugar, y durante los primeros años del siglo veinte se popularizaron las primeras pruebas estandarizadas sobretodo en Norteamérica con las pruebas de medición de la inteligencia y la consecuente difusión del concepto de "coeficiente intelectual" (IQ por su sigla en inglés) con la revisión que Lewis Termet hiciera del 'Binet test' en 1916. Desafortunadamente, durante esta época se cometieron diferentes tipos de excesos debido a que la mayoría de los instrumentos y pruebas 
Fundamentos para el desarrollo profesional de los profesores de inglés. 2a adición. Pasto: Editorial Universitaria - Universidad de Nariño. (361 pp.). 2015. ISBN 978-958-8609-98-0.

supuestamente estandarizadas carecían de validez y confiabilidad y de otros aspectos de validez física que hoy en día de son de importancia innegable.

La segunda etapa de las pruebas estandarizadas, todavía en el campo de la medición en psicología se difundió en el campo de la educación (conocido como psicometría). A partir de la investigación de campo contextualizada se enmendaron los errores y excesos cometidos en la etapa anterior producto de la aplicación de un positivismo exagerado. La medición en el campo de la personalidad se volvió más integral (a diferencia de la orientación individualizada en el pasado) mientras que la medición del aprendizaje en educación se encargaba de medir progresos con fines de mejoramiento del sistema en cuanto a aprendizaje con relación a un vasto conjunto de objetivos educacionales.

A principios de los años sesenta la estandarización de las pruebas en Norteamérica y Europa se había convertido en un fenómeno bastante aceptado en el ámbito educativo a tal punto de llegar a convertirse en una necesidad el hecho de conocer la elaboración de las pruebas mismas, su aplicación y su interpretación con el uso de los resultados para toma de decisiones y determinar cursos de acción.

\section{La competencia y actuación lingüísticas}

Generalmente para hablar de competencia en lenguas como en el caso del inglés hay que hacer referencia a las implicaciones que ha dejado la investigación científica sobre la adquisición de una segunda lengua y al aprendizaje de una lengua extranjera (Dulay, Burt, \& Krashen, 1982). Una muy buena parte de la literatura sobre la adquisición de una segunda lengua o el aprendizaje de una lengua extranjera se ha basado en las investigaciones de casos de niños y adultos que en forma individual aprendieron la lengua ya sea por razones culturales o instrumentales. Sin embargo, a menudo se hace la diferencia entre la competencia académica que tiene un estudiante y la competencia del idioma, pensando que las dos áreas no tienen mucha relación. 
Fundamentos para el desarrollo profesional de los profesores de inglés. 2a a Edición. Pasto: Editorial Universitaria - Universidad de Nariño. (361 pp.). 2015. ISBN 978-958-8609-98-0.

En este orden de ideas, las conclusiones de las observaciones que Hughes (2003) ha realizado sobre docentes y Hakuta, Butler, \& Daria (2000) sobre estudiantes en cuanto a la competencia del lenguaje permiten ver una correlación estrecha entre los resultados de las pruebas estandarizadas y la eficiencia académica del lenguaje por parte de los participantes. Es decir, la evaluación no es el único producto visible de la aplicación de las pruebas estandarizadas en un sistema educativo sino los productos colaterales de procesos como la lectura con propósito, la lectura intensiva, el análisis, la reflexión y la producción en una lengua a través de una prueba válida y confiable. Esto ha permitido que la gente se concentre en el desarrollo de los procesos verbales e intelectuales que se desprenden del ejercicio lingüístico a través del esfuerzo o reto cognitivo que permite una prueba estandarizada en un determinado periodo de aplicación.

La información sobre la habilidad lingüística de la gente es muy útil y, sobra decirlo, necesaria para determinar debilidades y fortalezas para la toma de decisiones al respecto. Sería muy difícil imaginar a profesores y estudiantes de una lengua extranjera sin un conocimiento y desarrollo por encima del básico de sus habilidades comunicativas. Se necesitaría información sobre cuál es el nivel de profesores y estudiantes con respecto a su competencia y actuación lingüísticas para determinar qué tan bien están realizando su función en términos de desarrollo pedagógico y del aprendizaje respectivamente. Para efectos de la enseñanza sería necesario proveer algún tipo de evaluación sobre cómo se están desarrollando los procesos académicos para el logro de los objetivos. Estos también se necesitarían para determinar qué niveles de logro tienen determinados grupos de estudiantes y así determinar los cursos de acción con respecto a contenidos, metodologías, materiales, profesores, capacitación, etc.

Las evaluaciones informales que a menudo realizan los docentes en instituciones educativas con los estudiantes son generalmente apropiadas, pero no lo suficiente al momento de evaluar un proceso dentro de un sistema de enseñanza en general o metodología y didáctica en particular. 
Fundamentos para el desarrollo profesional de los profesores de inglés. 2aa. Edición. Pasto: Editorial Universitaria - Universidad de Nariño. (361 pp.). 2015. ISBN 978-958-8609-98-0.

Sin dejar de considerar la posibilidad de sesgos hay que reconocer la necesidad de una 'unidad' común (estándar) con la cual evaluar y 'medir' para realizar comparaciones que tengan sentido, es decir, que sean válidas y confiables para la toma de decisiones en cuanto al cambio pertinente y contextual.

\section{La aplicación de pruebas para el diagnóstico del nivel de inglés}

En cuanto respecta al desarrollo y aplicación de técnicas de evaluación en los diferentes componentes y habilidades del inglés, Madsen (1983), presentó una importante cantidad de fórmulas para la elaboración de pruebas y la consecuente obtención de información adecuada para medir el aprendizaje. Además, Hughes (2003) había complementado el concepto de la elaboración de pruebas con criterios mínimos de comprensión de los principios de validez y de confiabilidad en los diferentes aspectos a evaluar, además de lo que implicaba la medición y evaluación del aprendizaje de aspectos tan esenciales como la comunicación en lengua extranjera. Sobre este tema, Underhill (1987) y Brown \& Yule, (1983) hicieron aportes importantes, sobre todo teniendo en cuenta las partes de fundamentación del proceso, y las técnicas de aplicación de la evaluación del aprendizaje.

En este campo específico se han propuesto muchos cursos de acción relacionados con la obtención de información válida y confiable en cuanto al aprendizaje se refiere. Fueron particularmente importantes los esfuerzos realizados por agencias internacionales como ETS (Educational Testing Service) en Princeton New Jersey, donde desde hace más de cincuenta años se ha aportado valiosa información sobre la medición de cada una de las habilidades lingüísticas a nivel mundial. La validez y confiabilidad de esta información radica en que proviene de pruebas de carácter estándar las cuales se aplican a millones de personas cada año en más de cien países y quienes se interesan en demostrar competencia lingüística y comunicativa en el idioma inglés. 
Fundamentos para el desarrollo profesional de los profesores de inglés. 2a a Edición. Pasto: Editorial Universitaria - Universidad de Nariño. (361 pp.). 2015. ISBN 978-958-8609-98-0.

Esta clase de información de diferente índole y origen ha sido objeto de estudio y análisis a nivel de grandes poblaciones de profesores $y$ estudiantes y sobre la cual existe actualización constante en cuanto a técnicas utilizadas en las pruebas en cada una de las habilidades lingüísticas. Mediante la aplicación de estudios de campo para validez y confiabilidad esta clase de pruebas ha ganado una amplia reputación a nivel mundial como diagnóstico del nivel de aprendizaje y adquisición de una lengua extranjera.

Un punto de innegable importancia es la consideración de que sea cual fuere la metodología utilizada en el proceso de enseñanza-aprendizaje del inglés (sea como segunda lengua o como lengua extranjera) las pruebas estandarizadas tendrían unos criterios consistentes con los objetivos y/o consideraciones generales y específicas de lo que es 'saber inglés' (uso de la lengua). Esto implica que las pruebas están generalmente dirigidas a las personas que han cumplido con un programa terminal de instrucción en la lengua objeto y por lo tanto están en condiciones de demostrar diferentes grados de dominio de la misma.

Desafortunadamente, en nuestro medio son muy escasos los estudios sobre la elaboración, aplicación e información de los análisis de resultados de esta clase de pruebas durante el proceso, es decir, de carácter secuencial formativo y sumativo, y su incidencia en el aprendizaje. Algunas se basan en formatos conocidos como las que existen comercialmente como es el caso de la serie de pruebas MAC: K-12 (Maculitis Assessment Program) las cuales determinan el nivel de competencia a través de varias etapas en áreas como fluidez oral, pronunciación, composición, vocabulario y escucha como también competencia oral. La otra serie de pruebas generalmente disponibles son las de OAEP (Orientation in American English Placement and Proficiency Test) las cuales también determinan el nivel de competencia en seis etapas desde estudiantes jóvenes hasta adultos en las diferentes áreas, habilidades y aspectos lingüísticos de la lengua extranjera. 
Fundamentos para el desarrollo profesional de los profesores de inglés. 2a a Edición. Pasto: Editorial Universitaria - Universidad de Nariño. (361 pp.). 2015. ISBN 978-958-8609-98-0.

\section{Las pruebas estandarizadas}

Una evaluación o medición estandarizada de tipo educativo es un intento formal por determinar el nivel de competencia de una persona con respecto a variables lingüísticas específicas como el conocimiento, y el uso. La diferencia entre lo que se hace diariamente en clase como evaluación informal y la evaluación mediante una prueba estandarizada radica en el significado del término 'formal', es decir, la forma sistemática y procedimental de evaluación de esta última. Un tipo informal o casual de evaluación, que es lo que el profesor hace en clase no podría equipararse a una evaluación formal que persigue información del sistema o subsistema de enseñanza y aprendizaje en tanto habilidades, conocimientos 0 aptitudes de una persona, en forma válida y confiable.

Para mucha gente, el termino prueba 'estandarizada' implica un tipo de evaluación por medio de un examen oral o escrito a nivel más sofisticado y formal que un examen o quiz. Las pruebas de Estado o pruebas 'Saber' o 'Saber PRO' conocidas hasta hace poco como exámenes del ICFES en el caso colombiano es un esfuerzo por estandarizar la evaluación del sistema educativo. Sin embargo, 'prueba' o 'examen' se pueden utilizar indistintamente haciendo claridad en el sentido de que el propósito de la estandarización es buscar evidencias sobre el estado de un determinado proceso de aprendizaje, dominio, habilidad, conocimiento, etc. que determinan de alguna manera la efectividad de un determinado sistema educativo, para la toma de decisiones que permitan mantenerlo o transformarlo mejorándolo.

A menudo se cree que una prueba estandarizada en lenguas es el reflejo fiel de un determinado estado del conocimiento o la habilidad lingüística de una persona. En realidad, los resultados de una prueba son la base sobre la cual se hacen inferencias en cuanto al nivel de conocimientos o habilidad de un determinado sujeto, pero no en su totalidad. De esta manera las pruebas estandarizadas constituyen un proceso de hacer inferencias apropiadas en forma sistemática basadas en la sistematización y estadística sobre el estado de desarrollo de una persona o grupo de 
Fundamentos para el desarrollo profesional de los profesores de inglés. 2aa. Edición. Pasto: Editorial Universitaria - Universidad de Nariño. (361 pp.). 2015. ISBN 978-958-8609-98-0.

personas con respecto a importantes variables lingüísticas como la habilidad lectora, el desarrollo de vocabulario, la destreza en la comunicación oral y la composición escrita.

Una prueba estandarizada es un mecanismo de evaluación y medición que se administra y se califica de una manera estándar o predeterminada y se compara con los resultados de un grupo de sujetos que constituyen la población denominado como el grupo 'norma', o sea, al grupo de referencia, con el cual se comparan los resultados de los grupos subsecuentes. Con esto como base se puede tener como principio que, si una persona obtuviera un resultado en el percentil 70, querría decir que el 70 por ciento de los casos del grupo 'norma', estaría por debajo de ese porcentaje y solo 30 por ciento aproximadamente por encima de ella. Estas pruebas estandarizadas suelen comprender lo que se conoce como pruebas de referencia a la norma (norm-referenced tests) ya que los resultados e inferencias que de ellas se desprenden se deben referenciar (o remitir para comparación) a una norma que tiene un grupo de personas que ya han tomado la prueba.

Además, una prueba estandarizada es aquella que se administra bajo condiciones controladas y se evalúa o mide en forma consistente o estándar. Está diseñada, desarrollada, supervisada y mejorada constantemente por especialistas que acumulan información sobre resultados de miles o millones de personas en todo el mundo durante el periodo de vida de la prueba. Con esto se garantiza que tanto la validez como la confiabilidad de la prueba fruto de la investigación de campo y de la aplicación estadística puedan proveer medidas muy cercanas a la realidad de lo que se evalúa. Sin embargo, generalmente la gente se desempeña en forma diferente, en distintas situaciones. Es bastante probable que una persona obtenga resultados distintos tomando la misma prueba en diferentes momentos, sin embargo, los resultados obtenidos así varíen estarán dentro de un determinado margen de error que se aplicaría para una importante parte de la población que haya tomado la prueba en diferentes circunstancias. 
Fundamentos para el desarrollo profesional de los profesores de inglés. 2a a Edición. Pasto: Editorial Universitaria - Universidad de Nariño. (361 pp.). 2015. ISBN 978-958-8609-98-0.

Este margen de error puede deberse también al azar y no necesariamente al nivel verdadero o real de la habilidad del examinado. El índice que generalmente se utiliza para describir el grado de precisión en una medida se denomina 'error estándar'. Por ejemplo, el caso de un error estándar de 14 en una prueba TOEFL de la década de los ochenta, más precisamente de los años 1987 a 1989 (de todos quienes tomaron la prueba en ese periodo) significaría que si el nivel real de una persona, es decir, el que obtendría si la prueba pudiera medir su habilidad de inglés con precisión perfecta, fuera de 560 , las posibilidades serían que el resultado estaría entre 546 y 574 (14 puntos por encima o por debajo de 560). De esta manera, todo lo que se derive de una prueba estándar también se consideraría estándar para efectos de su análisis, con la consecuente organización y sistematización de la información.

\section{La situación del inglés para las pruebas estandarizadas en Colombia}

En el momento de apertura, internacionalización y globalización que se encuentra el país en el contexto mundial es de imperiosa necesidad que se revise todo el sistema en el que se desenvuelve el proceso de enseñanza aprendizaje de una lengua extranjera como el inglés. Específicamente nos enfrentamos ante la necesidad de que los profesionales del Inglés en el futuro obtengan un nivel óptimo de uso de la lengua en sus diferentes habilidades: la escucha, el habla, la lectura y la composición escrita para quienes tengan que utilizar la lengua en forma comunicativa en casos de interacción directa ya sea con hablantes nativos del Inglés o con hablantes de otras lenguas que comparten el Inglés como medio de comunicación; el dominio del vocabulario y la comprensión de lectura para quienes tienen que manejar textos y literatura en el idioma en cuestión y además tienen que muy posiblemente hacerlos conocer a la comunidad científica y académica como comunicación directa y auténtica; y la composición escrita y la gramática para quienes como secretarias, ejecutivos, y científicos tienen que escribir textos en Inglés. 
Fundamentos para el desarrollo profesional de los profesores de inglés. 2a a Edición. Pasto: Editorial Universitaria - Universidad de Nariño. (361 pp.). 2015. ISBN 978-958-8609-98-0.

En primer lugar, es importante considerar que el aprendizaje del inglés como lengua extranjera en nuestro medio ha tenido el problema generalizado de su escaso uso práctico en diferentes contextos reales. En segundo lugar, teniendo en cuenta la importancia de saber inglés en este momento de apertura económica, educativa y científica y en el futuro inmediato, ha sido constante la preocupación por la consolidación de mecanismos que permitan evaluar la calidad de los estudiantes que egresan de las instituciones de educación básica, media y superior en cuanto al manejo de las habilidades del idioma inglés desde las diferentes especialidades y campos del saber incluida la formación en educación. Por otro lado, se conoce que los programas de inglés existentes en el país conducen a la formación de licenciados que engrosan las filas de profesores en educación básica (nivel de primaria y secundaria) y el grado de dominio de las habilidades lingüísticas de estos profesionales deja mucho que desear según el diagnóstico realizado por el British Council (2006) realizado en establecimientos de bachillerato del municipio de Pasto donde el nivel de los docentes estaba lejos del deseado por el Ministerio de Educación Nacional. Esto se agrava aún más cuando se determina que el nivel de lengua de los docentes no llegaría a los niveles exigidos para enseñar la lengua afectando de esta manera los niveles de competencia de los estudiantes que terminan la educación secundaria y que consecuentemente inician la educación superior.

Por consiguiente, se podría inferir que el nivel de inglés que se requiere para los futuros profesionales: secretarias, ejecutivos, ingenieros, abogados, médicos, etc. no se cubriría con el desarrollo que se da a nivel de lengua en las diferentes habilidades al término del proceso de educación formal. Por esta razón la gente tendría que recurrir a cursos especiales y/o programas intensivos de inglés ya sea dentro o fuera del país, especialmente en el nivel privado.

Este problema del bajo nivel de inglés de los docentes que tienen a su cargo la enseñanza del inglés en la educación básica tiene implicaciones en el área de capacitación y actualización de conocimientos y estrategias de enseñanza y aprendizaje. Así mismo se hace más notorio cuando son los 
Fundamentos para el desarrollo profesional de los profesores de inglés. 2aa. Edición. Pasto: Editorial Universitaria - Universidad de Nariño. (361 pp.). 2015. ISBN 978-958-8609-98-0.

estudiantes quienes tendrían dificultados para ajustarse a las demandas de este conocimiento en la universidad, sin contar con los obstáculos encontrados el acceso a becas internacionales en un medio académico cada vez más competitivo donde el conocimiento y manejo de la lengua extrajera es de una estricta exigencia. Así pues, muchas de las oportunidades que tienen los futuros profesionales de salir a realizar especializaciones en los diferentes campos se perderían por la carencia del conocimiento y manejo adecuados del inglés.

\section{Impacto de las pruebas estandarizadas en educación en lenguas}

El impacto que se puede vislumbrar en un proyecto de uso y aplicación de las pruebas estandarizadas es en realidad un complemento entre los aspectos científico, pedagógico y tecnológico. En primer lugar, el proceso de estandarización implicaría un camino hacia la investigación en evaluación de tipo válido, confiable y además práctico puesto que los objetivos son en últimas los que se evalúan para verificar que los niveles de aprendizaje de la lengua sean los óptimos. Así mismo, la base pedagógica se enriquecería por las implicaciones de retroalimentación que tendría el sistema educativo mediante la investigación de campo en evaluación. Por otro lado, el uso de las pruebas a través de las nuevas tecnologías, de la informática y de la sistematización que agilizarían el acceso a las pruebas y por ende a los resultados y análisis de los mismos y su aplicación en la verificación y diagnóstico de procesos como la revisión tanto de objetivos, contenidos como criterios de enseñanza y aprendizaje. Esto permitiría que el proceso de evaluación se consolide como un mecanismo ágil, consistente y de fácil acceso tanto a nivel de realización de las pruebas como de procesamiento estadístico de la información en retroalimentación de programas.

\section{Relación docencia-evaluación}

El hecho de contar con un proceso de evaluación de la lengua extranjera mediante pruebas estandarizadas durante el proceso de enseñanzaaprendizaje implicaría enfocar los contenidos programáticos hacia aquellos aspectos que puedan ser verificados en su desarrollo. Una apertura a lo 
Fundamentos para el desarrollo profesional de los profesores de inglés. 2a a Edición. Pasto: Editorial Universitaria - Universidad de Nariño. (361 pp.). 2015. ISBN 978-958-8609-98-0.

verificable en la práctica de un proceso de aprendizaje de una lengua extrajera como competencia y actuación lingüísticas determinaría el redireccionamiento tanto de las metas, objetivos y en general los currículos de lengua extranjera. Además, todo este proceso al estar sistematizado por niveles y habilidades comprobables mediante las pruebas serviría como base para realizar la retroalimentación al mismo proceso de aprendizaje teniendo en cuenta los contextos, las necesidades y debilidades y las fortalezas y potencialidades, trabajando sobre las primeras y aprovechando las últimas.

En el primer caso, los esfuerzos tanto de diseñadores, instructores, evaluadores e investigadores estarían dirigidos a aquellos aspectos programáticos (metodología, contenidos, materiales, etc.) que conlleven al desarrollo de los conocimientos y las habilidades expresados en los objetivos de los programas a través del énfasis de la evaluación mediante pruebas estandarizadas. En cuanto a la parte investigativa, el docente podrá, basado en los resultados del aprendizaje logrado, investigar y experimentar con diferentes tipos de metodologías, técnicas, estrategias, contenidos, materiales, etc. en la seguridad de que la evaluación tendrá una consistencia basada en los objetivos de un determinado plan. Esta parte es importante por cuanto la misma investigación se fortalecería al poder aplicar sistemáticamente las pruebas sobre la base del seguimiento de los programas académicos. Este proceso de tipo pedagógico investigativo se constituiría en un soporte de la docencia al aportar instrumentos válidos y confiables con los cuales se pueda medir el grado de aprendizaje de la lengua extranjera.

\section{Relación evaluación, enseñanza e investigación}

Existe a nivel práctico una relación entre la enseñanza y el hecho de evaluar el aprendizaje que la enseñanza pretende como producto. Se conoce como sentido común que el docente se puede dar cuenta que, si se realiza un buen trabajo instruccional, los estudiantes generalmente obtendrán buenos resultados en las pruebas. Este es el otro lado de la moneda que es usualmente menos entendido, es decir, por un lado, la 
Fundamentos para el desarrollo profesional de los profesores de inglés. 2a a Edición. Pasto: Editorial Universitaria - Universidad de Nariño. (361 pp.). 2015. ISBN 978-958-8609-98-0.

forma en que un docente evalúa o mide el aprendizaje de sus estudiantes y por el otro la forma cómo aplica ese conocimiento práctico en el mejoramiento de la práctica docente -la forma como se utilizan los resultados de una prueba que puede afectar positivamente el proceso de enseñanza y aprendizaje.

Consecuentemente, la consideración de la relación entre la forma de evaluar y la forma de enseñar sería muy importante en el sentido de que si se entiende adecuadamente el proceso cuantitativo y cualitativo de la evaluación este puede llevar a un importante incremento de la efectividad en los recursos de la competencia pedagógica del docente. Esto ha llevado a considerar las pruebas estandarizadas no en el sentido de que solo medir o evaluar apropiadamente mejora la enseñanza sino el hecho de que formas efectivas de enseñar tendrían que estar relacionadas con formas más efectivas de evaluar. Por consiguiente, no se está hablando solo de técnicas de evaluación o de técnicas de realización de pruebas diagnósticas para medición de habilidades sino de la evaluación educativa como concepto cualitativo e integral del mismo sistema.

\section{Pruebas estandarizadas para evaluar el nivel de inglés}

Algunas pruebas estandarizadas que miden el nivel de inglés como el TOEFL (Test of English as a Foreign Language) de Educational Testing Service, los de la Universidad de Cambridge U.K. (KET, PET, FCE, CAE, y CPE), y los de la Universidad de Oxford como el OPT (Oxford Placement Test), entre otros, sirven para evaluar el conocimiento y manejo de la lengua extranjera de las personas cuya lengua nativa no es el inglés. Esta evaluación se hace en varios aspectos como vocabulario, gramática, lectura, comprensión de escucha, producción oral, composición escrita, entre otras. La mayoría de las personas que toman esta clase de pruebas son estudiantes que tienen como meta estudiar a nivel superior en un país angloparlante. De la misma manera, muchas agencias e instituciones educativas en diferentes países utilizan pruebas estandarizadas para la evaluación del nivel de inglés como segunda lengua y como lengua extranjera tanto en docentes como en estudiantes. Por ejemplo, más de 
Fundamentos para el desarrollo profesional de los profesores de inglés. 2aa. Edición. Pasto: Editorial Universitaria - Universidad de Nariño. (361 pp.). 2015. ISBN 978-958-8609-98-0.

2500 universidades y facultades de los Estados Unidos, Canadá y otros países de Europa y Australia exigen a los candidatos no nativos del inglés tomar pruebas como el TOEFL; la serie de pruebas de Cambridge como el First Certificate in English u otras de tipo estandarizado como requisito de admisión. Además, el inglés como lengua extranjera está siendo exigido por un número cada vez más creciente de universidades del mundo como requisito de grado en programas de pregrado y consecuentemente también de postgrado.

\section{Las pruebas de Oxford de ubicación (OPT)}

Las Pruebas Oxford de Ubicación (OPT, por su sigla en inglés) son el resultado de muchos años de investigación sobre los problemas teóricos y prácticos que intervienen en la producción/diseño y aplicación de una prueba de ubicación/evaluación que sea válida y confiable, y además ágil, económica y fácil de administrar.

La prueba en papel (Allan, 2004) incluye dos secciones, una de escucha (discriminación y comprensión) y otra de gramática (lectura, vocabulario), que ponen a prueba los conocimientos lingüísticos, así como el conocimiento de inglés como sistema. En conjunto, los resultados totales de las dos secciones proporcionan rangos que se correlacionan altamente con otras pruebas estandarizadas de igual nivel de elaboración en confiabilidad y validez a nivel mundial. Las pruebas OPT han sido objeto de estudio, análisis y mejoramiento durante los tres últimos años de ensayos prácticos en instituciones de enseñanza de idiomas en todo el mundo. Todos los ítems han sido previamente probados en hablantes nativos de inglés y se han aplicado en su formato final en una amplia gama de contextos con ambos grupos, monolingües y multilingües en todos los niveles de educación desde el elemental hasta el alto-avanzado.

Dentro de esta gama de niveles, desde elemental hasta avanzado, las Pruebas Oxford de Ubicación han arrojado siempre una distribución de puntajes que reflejan los diferentes niveles en el grupo sometido a 
Fundamentos para el desarrollo profesional de los profesores de inglés. 2a a Edición. Pasto: Editorial Universitaria - Universidad de Nariño. (361 pp.). 2015. ISBN 978-958-8609-98-0.

ensayo. Se puede completar toda la prueba en aproximadamente una hora (alrededor de 10 minutos para escucha y 50 para gramática), y los resultados han sido un reflejo objetivo y preciso del nivel de manejo del sistema gramatical del inglés por el profesor y de otra serie de competencias claves. La prueba se puede utilizar en forma eficaz y confiable y ha sido clasificada dentro de los seis niveles que maneja el Marco Común Europeo de referencia para las Lenguas (MCERL o CEFRL por su sigla en inglés).

Esta es una característica importante de la reciente edición de la Prueba Oxford de Ubicación la cual ha sido integrada dentro del estándar del Marco Común Europeo para las Lenguas (ver tabla 1, abajo) a través de una serie de correlaciones directas. La vinculación de los puntajes OPT con los niveles del MCE ha hecho posible que los resultados OPT estén relacionados con los niveles correspondientes a las pruebas de Cambridge Main Suite, así como a pruebas IELTS, BEC y del CELS. Los resultados de las pruebas OPT también pueden correlacionarse, aunque no al mismo nivel de significación estadística, con el TOEFL y TOEIC.

El siguiente es un ejemplo (no necesariamente de casos reales) de 8 sujetos con los siguientes resultados en la prueba OPT tanto en Escucha como en Gramática.

Tabla 1. Ejemplo de ubicación de la prueba OPT
\begin{tabular}{|l|c|c|c|c|}
\hline NOMBRE & LISTENING & GRAMMAR & TOTAL & NIVEL MCE \\
\hline Carlos Aguirre & 75 & 81 & 156 & C1 \\
\hline Mauro López & 63 & 68 & 131 & B1 \\
\hline Juan Carreño & 70 & 74 & 144 & B2 \\
\hline Carmen Cerón & 45 & 61 & 106 & A2 \\
\hline Jenny Gómez & 72 & 98 & 170 & C2 \\
\hline José Álvarez & 51 & 62 & 113 & A2 \\
\hline Silvia Castillo & 39 & 45 & 84 & $<$ A1 \\
\hline
\end{tabular}


Fundamentos para el desarrollo profesional de los profesores de inglés. 2a a Edición. Pasto: Editorial Universitaria - Universidad de Nariño. (361 pp.). 2015. ISBN 978-958-8609-98-0.

Los rangos de equivalencias del OPT con respecto a los criterios del Marco Común Europeo son como se muestran en la tabla 2 (abajo)haciendo claridad que $<\mathrm{A} 1$ (menor que el nivel A1) no constituye en sí un nivel determinado, sino que significa que está por debajo de los rangos establecidos.

Tabla 2. Rangos de puntajes OPT en papel y equivalencia en el MCE

\begin{tabular}{|c|c|}
\hline Rangos de Puntajes OPT & Niveles MCE \\
\hline $170-189$ & C2 \\
\hline $150-169$ & C1 \\
\hline $135-149$ & B2 \\
\hline $120-134$ & B1 \\
\hline $105-119$ & A2 \\
\hline $90-104$ & A1 \\
\hline
\end{tabular}

Sin embargo, en forma más reciente las pruebas ya han dejado de lado el papel y lápiz como mecanismo físico del instrumento y se han integrado con las Nuevas Tecnologías de la Información y la Comunicación (NTIC) para a través de Internet realizar el proceso en línea (on-line), es decir en tiempo real y con la obtención inmediata de resultados. Ya se han hecho muy populares por su fácil y acceso más conveniente en el mundo los TOEFL IBT (por sus siglas en inglés InternetBased Testing), introducido desde el 2005 o las de ubicación de Oxford con el ya conocido OOPT (Oxford On-line Placement Test) que incorpora lo que se denomina Computer-Adaptive Language Testing que permite la selección de la secuenciación de preguntas según el nivel de dificultad que responda la persona y así determinar su nivel de inglés en espacio de aproximadamente una hora. Los resultados están dados dentro del Marco Común Europeo (MCE).

En aproximadamente 45 preguntas que incluyen dos aspectos de evaluación, uso de la lengua y comprensión de escucha la prueba arroja los resultados en los siguientes rangos del MCE. 
Fundamentos para el desarrollo profesional de los profesores de inglés. 2a a Edición. Pasto: Editorial Universitaria - Universidad de Nariño. (361 pp.). 2015. ISBN 978-958-8609-98-0.

Tabla 3. Rangos de puntajes OOPT (Online) equivalencia en el MCE
\begin{tabular}{|c|c|}
\hline Rangos de Puntajes OOPT & Niveles MCE \\
\hline $100-$ & C2 \\
\hline $80-99$ & C1 \\
\hline $60-79$ & B2 \\
\hline $40-59$ & B1 \\
\hline $20-39$ & A2 \\
\hline $0-19$ & A1 \\
\hline
\end{tabular}

Este tipo de pruebas estandarizadas son la que en el momento se han utilizado por el Ministerio de Educación Nacional en el Proyecto de Fortalecimiento de Programas de Licenciatura en Lengua extranjera, inglés, en una fase diagnóstica a estudiantes y profesores de programas de licenciatura en idiomas dentro del Programa de Fortalecimiento al Desarrollo de Competencias en Lenguas Extranjeras (PFDCLE) como ahora se denomina el anterior Programa de Bilingüismo (MEN, 2012). Haría falta la adopción de un sistema estandarizado de este tipo para las pruebas 'Saber 5', 'Saber 11 ' y 'Saber Pro' como referente internacional comparable.

\section{El Marco Común Europeo de referencia para las lenguas (MCE)}

El Marco Común Europeo de referencia para las lenguas establece una serie de seis niveles $(A 1, A 2, B 1, B 2, C 1, C 2)$, para todas las lenguas a partir de los cuales se favorece la comparación u homologación de los distintos títulos emitidos por las entidades certificadas. El proyecto fue propuesto en un congreso internacional celebrado en Suiza en noviembre de 1991 y desarrollado por el Consejo de Europa.

El Ministerio de Educación Nacional en su afán de articularse en los procesos de internacionalización, intercambio educativo y cultural recientemente ha adoptado la normatividad estándar que establece el Marco Común Europeo de referencia para las lenguas. Este marco 
Fundamentos para el desarrollo profesional de los profesores de inglés. 2a a Edición. Pasto: Editorial Universitaria - Universidad de Nariño. (361 pp.). 2015. ISBN 978-958-8609-98-0.

internacional que se interesa en el aprendizaje, la enseñanza y la evaluación de las lenguas es un estándar que pretende servir de patrón internacional para medir el nivel de conocimiento varias lenguas como el inglés. Para este efecto el Ministerio de Educación Nacional de Colombia ha establecido que el nivel de competencia exigido para un docente de inglés en educación básica y medía sea el de B2 según el MCE, es decir, un nivel 'superior' de usuario independiente o intermedio alto (ver tabla 3), el mismo que se exige para un egresado universitario y C1 para un egresado en lenguas extranjeras

\section{Configuración inicial de niveles}

Recopilando información acerca de los distintos niveles propuestos por otras entidades y autores, los encargados del proyecto observaron que el número más adecuado de niveles para el aprendizaje, enseñanza y evaluación de las lenguas era de seis. Estos no necesariamente coinciden con los niveles clásicos de básico, intermedio y avanzado, al estar situados por encima o por debajo de ellos, respectivamente. Con base en esto, se elaboró una distinción inicial en tres bloques amplios, cada bloque se divide en otros dos niveles más restrictivos.

Tabla 4: Bloques, usuarios, niveles y significados del MCE
\begin{tabular}{|l|l|}
\hline BLOQUE A: Usuario Básico & SIGNIFICADO \\
\hline Nivel A1: Ingreso & Principiante \\
\hline Nivel A2: Básico & Elemental \\
\hline BLOQUE B: Usuario independiente & \\
\hline Nivel B1: Límite & Intermedio \\
\hline Nivel B2: Superior & Intermedio Alto \\
\hline BLOQUE C: Usuario competente & \\
\hline Nivel C1: Dominio operativo eficaz & Avanzado \\
\hline Nivel C2: Maestría de la lengua & Avanzado Alto \\
\hline
\end{tabular}

\section{Configuración final}


Fundamentos para el desarrollo profesional de los profesores de inglés. 2a adición. Pasto: Editorial Universitaria - Universidad de Nariño. (361 pp.). 2015. ISBN 978-958-8609-98-0.

El desarrollo posterior del MCE permite la simplificación del esquema anterior al siguiente, que facilita en gran medida la comprensibilidad a los usuarios de la información, presentando una tabla de escala global para cada uno de los seis niveles.

- Nivel A1: Se adquiere cuando el sujeto es capaz de comprender y utilizar expresiones cotidianas de uso muy frecuente, así como frases sencillas destinadas a satisfacer necesidades de tipo inmediato; cuando puede presentarse a sí mismo y a otros, pedir y dar información personal básica sobre su domicilio, sus pertenencias y las personas que conoce y cuando puede relacionarse de forma elemental siempre que su interlocutor hable despacio y con claridad y esté dispuesto a cooperar.

- Nivel A2: Se adquiere cuando el sujeto es capaz de comprender frases y expresiones de uso frecuente relacionadas con áreas de experiencia que le son especialmente relevantes (información básica sobre sí mismo y su familia, compras, lugares de interés, ocupaciones, etc.); cuando sabe comunicarse a la hora de llevar a cabo tareas simples y cotidianas que no requieran más que intercambios sencillos y directos de información sobre cuestiones que le son conocidas o habituales y cuando sabe describir en términos sencillos aspectos de su pasado y su entorno así como cuestiones relacionadas con sus necesidades inmediatas.

- Nivel B1: Se adquiere cuando el sujeto es capaz de comprender los puntos principales de textos claros y en lengua estándar si tratan sobre cuestiones que le son conocidas, ya sea en situaciones de trabajo, de estudio o de ocio; cuando sabe desenvolverse en la mayor parte de las situaciones que pueden surgir durante un viaje por zonas donde se utiliza la lengua; cuando es capaz de producir textos sencillos y coherentes (discurso oral o escrito) sobre temas que le son familiares o en los que tiene un interés personal y cuando puede describir experiencias, acontecimientos, deseos y aspiraciones, así como justificar brevemente sus opiniones o explicar sus planes. 
Fundamentos para el desarrollo profesional de los profesores de inglés. 2a adición. Pasto: Editorial Universitaria - Universidad de Nariño. (361 pp.). 2015. ISBN 978-958-8609-98-0.

- Nivel B2: Se adquiere cuando el sujeto es capaz de entender las ideas principales de textos complejos que traten de temas tanto concretos como abstractos, incluso si son de carácter técnico siempre que estén dentro de su campo de especialización; cuando puede relacionarse con hablantes nativos con un grado suficiente de fluidez y naturalidad de modo que la comunicación se realice sin esfuerzo por parte de ninguno de los interlocutores y cuando puede producir textos claros y detallados sobre temas diversos así como defender un punto de vista sobre temas generales indicando los pros y los contras de las distintas opciones.

- Nivel C1: Se adquiere cuando el sujeto es capaz de comprender una amplia variedad de textos extensos y con cierto nivel de exigencia, así como reconocer en ellos sentidos implícitos; cuando sabe expresarse de forma fluida y espontánea sin muestras muy evidentes de esfuerzo para encontrar la expresión adecuada; cuando puede hacer un uso flexible y efectivo del idioma para fines sociales, académicos y profesionales y cuando puede producir textos claros, bien estructurados y detallados sobre temas de cierta complejidad, mostrando un uso correcto de los mecanismos de organización, articulación y cohesión del texto.

- Nivel C2: Se adquiere cuando el sujeto es capaz de comprender con facilidad prácticamente todo lo que oye o lee; cuando sabe reconstruir la información y los argumentos procedentes de diversas fuentes, ya sean en lengua hablada o escrita, y presentarlos de manera coherente y resumida y cuando puede expresarse espontáneamente, con gran fluidez y con un grado de precisión que le permite diferenciar pequeños matices de significado incluso en situaciones de mayor complejidad. 
Fundamentos para el desarrollo profesional de los profesores de inglés. 2a. Edición. Pasto: Editorial Universitaria - Universidad de Nariño. (361 pp.). 2015. ISBN 978-958-8609-98-0.

A continuación, se puede observar los seis niveles de MCE en cuanto a tres áreas de competencia lingüística y comunicativa para escucha, lectura, producción oral y producción escrita.

Tabla 5: Habilidades lingüísticas y comunicativas desarrolladas en cada nivel según el MCE (tomado de la traducción realizada por el Instituto Cervantes, 2002)

\begin{tabular}{|c|c|c|c|c|c|}
\hline \multirow[t]{2}{*}{ Niveles } & \multicolumn{2}{|c|}{ Comprender } & \multicolumn{2}{|c|}{ Hablar } & \multirow{2}{*}{$\begin{array}{c}\text { Escribir } \\
\text { Expresión escrita }\end{array}$} \\
\hline & $\begin{array}{c}\text { Comprensión } \\
\text { auditiva }\end{array}$ & $\begin{array}{c}\text { Comprensión de } \\
\text { lectura }\end{array}$ & Interacción oral & Expresión oral & \\
\hline $\mathrm{A} 1$ & \begin{tabular}{|l|} 
Reconozco \\
palabras y \\
expresiones muy \\
básicas que se \\
usan \\
habitualmente, \\
relativas a mí \\
mismo, a mi \\
familia y a mi \\
entorno \\
inmediato \\
cuando se habla \\
despacio y con \\
claridad.
\end{tabular} & \begin{tabular}{|l|} 
Comprendo \\
palabras y \\
nombres \\
conocidos y frases \\
muy sencillas, por \\
ejemplo las que \\
hay en letreros, \\
carteles y \\
catálogos.
\end{tabular} & \begin{tabular}{||l|} 
Puedo participar en \\
una conversación de \\
forma sencilla \\
siempre que la otra \\
persona esté \\
dispuesta a repetir \\
lo que ha dicho o a \\
decirlo con otras \\
palabras y a una \\
velocidad más lenta \\
y me ayude a \\
formular lo que \\
intento decir. \\
Planteo y contesto \\
preguntas sencillas \\
sobre temas de \\
necesidad inmediata \\
o asuntos muy \\
habituales. \\
\end{tabular} & \begin{tabular}{|l|} 
Utilizo \\
expresiones y \\
frases sencillas \\
para describir \\
el lugar donde \\
vivo y las \\
personas que \\
conozco.
\end{tabular} & \begin{tabular}{|l|} 
Soy capaz de \\
escribir postales \\
cortas y sencillas, \\
por ejemplo para \\
enviar \\
felicitaciones. Sé \\
rellenar \\
formularios con \\
datos personales, \\
por ejemplo mi \\
nombre, mi \\
nacionalidad y mi \\
dirección en el \\
formulario del \\
registro de un \\
hotel.
\end{tabular} \\
\hline$A 2$ & \begin{tabular}{||l|} 
Comprendo \\
frases y el \\
vocabulario más \\
habitual sobre \\
temas de interés \\
personal \\
(información \\
personal y \\
familiar muy \\
básica, compras, \\
lugar de \\
residencia, \\
empleo). Soy \\
capaz de captar \\
la idea principal \\
de avisos y \\
mensajes breves, \\
claros y sencillos.
\end{tabular} & \begin{tabular}{|l|} 
Soy capaz de leer \\
textos muy breves \\
y sencillos. Sé \\
encontrar \\
información \\
específica y \\
predecible en \\
escritos sencillos y \\
cotidianos como \\
anuncios \\
publicitarios, \\
prospectos, menús \\
y horarios y \\
comprendo cartas \\
personales breves \\
y sencillas.
\end{tabular} & \begin{tabular}{||l|} 
Puedo comunicarme \\
en tareas sencillas y \\
habituales que \\
requieren un \\
intercambio simple \\
y directo de \\
información sobre \\
actividades y \\
asuntos cotidianos. \\
Soy capaz de realizar \\
intercambios \\
sociales muy breves, \\
aunque, por lo \\
general, no puedo \\
comprender lo \\
suficiente como \\
para mantener la \\
conversación por mí \\
mismo.
\end{tabular} & \begin{tabular}{|l|} 
Utilizó una \\
serie de \\
expresiones y \\
frases para \\
describir con \\
términos \\
sencillos a mi \\
familia y otras \\
personas, mis \\
condiciones de \\
vida, mi origen \\
educativo y mi \\
trabajo actual \\
o el último que \\
tuve.
\end{tabular} & \begin{tabular}{|l|} 
Soy capaz de \\
escribir notas y \\
mensajes breves y \\
sencillos relativos \\
a mis necesidades \\
inmediatas. Puedo \\
escribir cartas \\
personales muy \\
sencillas, por \\
ejemplo \\
agradeciendo algo \\
a alguien.
\end{tabular} \\
\hline B1 & \begin{tabular}{|l|} 
Comprendo las \\
ideas principales \\
cuando el \\
discurso es claro \\
y normal y se \\
tratan asuntos \\
cotidianos que \\
tienen lugar en el \\
trabajo, en la \\
\end{tabular} & \begin{tabular}{|l|} 
Comprendo textos \\
redactados en una \\
lengua de uso \\
habitual y \\
cotidiano o \\
relacionada con el \\
trabajo. \\
Comprendo la \\
descripción de \\
\end{tabular} & \begin{tabular}{|l|} 
Sé desenvolverme \\
en casi todas las \\
situaciones que se \\
me presentan \\
cuando viajo donde \\
se habla esa lengua. \\
Puedo participar \\
espontáneamente \\
en una conversación
\end{tabular} & \begin{tabular}{|l} 
Sé enlazar \\
frases de \\
forma sencilla \\
con el fin de \\
describir \\
experiencias y \\
hechos, mis \\
sueños, \\
esperanzas y \\
\end{tabular} & \begin{tabular}{|l} 
Soy capaz de \\
escribir textos \\
sencillos y bien \\
enlazados sobre \\
temas que me son \\
conocidos o de \\
interés personal. \\
Puedo escribir \\
cartas personales \\
\end{tabular} \\
\hline
\end{tabular}


Fundamentos para el desarrollo profesional de los profesores de inglés. 2a. Edición. Pasto: Editorial Universitaria - Universidad de Nariño. (361 pp.). 2015. ISBN 978-958-8609-98-0.

\begin{tabular}{|c|c|c|c|c|c|}
\hline & $\begin{array}{l}\text { escuela, durante } \\
\text { el tiempo de } \\
\text { ocio, etc. } \\
\text { Comprendo la } \\
\text { idea principal de } \\
\text { muchos } \\
\text { programas de } \\
\text { radio o televisión } \\
\text { que tratan temas } \\
\text { actuales o } \\
\text { asuntos de } \\
\text { interés personal } \\
\text { o profesional, } \\
\text { cuando la } \\
\text { articulación es } \\
\text { relativamente } \\
\text { lenta y clara. } \\
\end{array}$ & $\begin{array}{l}\text { acontecimientos, } \\
\text { sentimientos y } \\
\text { deseos en cartas } \\
\text { personales. }\end{array}$ & $\begin{array}{l}\text { que trate temas } \\
\text { cotidianos de } \\
\text { interés personal o } \\
\text { que sean } \\
\text { pertinentes para la } \\
\text { vida diaria (por } \\
\text { ejemplo, familia, } \\
\text { aficiones, trabajo, } \\
\text { viajes y } \\
\text { acontecimientos } \\
\text { actuales). }\end{array}$ & \begin{tabular}{||l|} 
ambiciones. \\
Puedo explicar \\
y justificar \\
brevemente \\
mis opiniones \\
y proyectos. Sé \\
narrar una \\
historia o \\
relato, la \\
trama de un \\
libro o película \\
y puedo \\
describir mis \\
reacciones.
\end{tabular} & $\begin{array}{l}\text { que describen } \\
\text { experiencias e } \\
\text { impresiones. }\end{array}$ \\
\hline B2 & $\begin{array}{l}\text { Comprendo } \\
\text { discursos y } \\
\text { conferencias } \\
\text { extensos e } \\
\text { incluso sigo líneas } \\
\text { argumentales } \\
\text { complejas } \\
\text { siempre que el } \\
\text { tema sea } \\
\text { relativamente } \\
\text { conocido. } \\
\text { Comprendo casi } \\
\text { todas las noticias } \\
\text { de la televisión y } \\
\text { los programas } \\
\text { sobre temas } \\
\text { actuales. } \\
\text { Comprendo la } \\
\text { mayoría de las } \\
\text { películas en las } \\
\text { que se habla en } \\
\text { un nivel de } \\
\text { lengua estándar. }\end{array}$ & \begin{tabular}{|l|} 
Soy capaz de leer \\
artículos e \\
informes relativos \\
a problemas \\
contemporáneos \\
en los que los \\
autores adoptan \\
posturas o puntos \\
de vista concretos. \\
Comprendo la \\
prosa literaria \\
contemporánea.
\end{tabular} & \begin{tabular}{|l} 
Puedo participar en \\
una conversación \\
con cierta fluidez y \\
espontaneidad, lo \\
que posibilita la \\
comunicación \\
normal con \\
hablantes nativos. \\
Puedo tomar parte \\
activa en debates \\
desarrollados en \\
situaciones \\
cotidianas \\
explicando y \\
defendiendo mis \\
puntos de vista.
\end{tabular} & \begin{tabular}{|l|} 
Presento \\
descripciones \\
claras y \\
detalladas de \\
una amplia \\
serie de temas \\
relacionados \\
con mi \\
especialidad. \\
Sé explicar un \\
punto de vista \\
sobre un tema \\
exponiendo las \\
ventajas y los \\
inconvenientes \\
de varias \\
opciones.
\end{tabular} & $\begin{array}{l}\text { Soy capaz de } \\
\text { escribir textos } \\
\text { claros y detallados } \\
\text { sobre una amplia } \\
\text { serie de temas } \\
\text { relacionados con } \\
\text { mis intereses. } \\
\text { Puedo escribir } \\
\text { redacciones o } \\
\text { informes } \\
\text { transmitiendo } \\
\text { información o } \\
\text { proponiendo } \\
\text { motivos que } \\
\text { apoyen o refuten } \\
\text { un punto de vista } \\
\text { concreto. Sé } \\
\text { escribir cartas que } \\
\text { destacan la } \\
\text { importancia que } \\
\text { le doy a } \\
\text { determinados } \\
\text { hechos y } \\
\text { experiencias. }\end{array}$ \\
\hline $\mathrm{C} 1$ & \begin{tabular}{|l} 
Comprendo \\
discursos \\
extensos incluso \\
cuando no están \\
estructurados \\
con claridad y \\
cuando las \\
relaciones están \\
sólo implícitas y \\
no se señalan \\
explícitamente. \\
Comprendo sin \\
mucho esfuerzo \\
los programas de \\
televisión y las \\
películas.
\end{tabular} & \begin{tabular}{|l|} 
Comprendo textos \\
largos y complejos \\
de carácter \\
literario o basados \\
en hechos, \\
apreciando \\
distinciones de \\
estilo. \\
Comprendo \\
artículos \\
especializados e \\
instrucciones \\
técnicas largas, \\
aunque no se \\
relacionen con mi \\
especialidad.
\end{tabular} & \begin{tabular}{|l|} 
Me expreso con \\
fluidez y \\
espontaneidad sin \\
tener que buscar de \\
forma muy evidente \\
las expresiones \\
adecuadas. Utilizo el \\
lenguaje con \\
flexibilidad y eficacia \\
para fines sociales y \\
profesionales. \\
Formulo ideas y \\
opiniones con \\
precisión y relaciono \\
mis intervenciones \\
hábilmente con las \\
de otros hablantes.
\end{tabular} & \begin{tabular}{|l|} 
Presento \\
descripciones \\
claras y \\
detalladas \\
sobre temas \\
complejos que \\
incluyen otros \\
temas, \\
desarrollando \\
ideas \\
concretas y \\
terminando \\
con una \\
conclusión \\
apropiada.
\end{tabular} & \begin{tabular}{|l} 
Soy capaz de \\
expresarme en \\
textos claros y \\
bien \\
estructurados \\
exponiendo \\
puntos de vista \\
con cierta \\
extensión. Puedo \\
escribir sobre \\
temas complejos \\
en cartas, \\
redacciones o \\
informes \\
resaltando lo que \\
considero que son \\
aspectos \\
importantes. \\
Selecciono el \\
estilo apropiado
\end{tabular} \\
\hline
\end{tabular}


Fundamentos para el desarrollo profesional de los profesores de inglés. 2a. Edición. Pasto: Editorial Universitaria - Universidad de Nariño. (361 pp.). 2015. ISBN 978-958-8609-98-0.

\begin{tabular}{|c|c|c|c|c|c|}
\hline & & & & & $\begin{array}{l}\text { para los lectores a } \\
\text { los que van } \\
\text { dirigidos mis } \\
\text { escritos. }\end{array}$ \\
\hline C2 & $\begin{array}{l}\text { No tengo ninguna } \\
\text { dificultad para } \\
\text { comprender } \\
\text { cualquier tipo de } \\
\text { lengua hablada, } \\
\text { tanto en } \\
\text { conversaciones } \\
\text { en vivo como en } \\
\text { discursos } \\
\text { retransmitidos, } \\
\text { aunque se } \\
\text { produzcan a una } \\
\text { velocidad de } \\
\text { hablante nativo, } \\
\text { siempre que } \\
\text { tenga tiempo } \\
\text { para } \\
\text { familiarizarme } \\
\text { con el acento. }\end{array}$ & $\begin{array}{l}\text { Soy capaz de leer } \\
\text { con facilidad } \\
\text { prácticamente } \\
\text { todas las formas } \\
\text { de lengua escrita, } \\
\text { incluyendo textos } \\
\text { abstractos } \\
\text { estructural o } \\
\text { lingüísticamente } \\
\text { complejos como, } \\
\text { por ejemplo, } \\
\text { manuales, } \\
\text { artículos } \\
\text { especializados y } \\
\text { obras literarias. }\end{array}$ & $\begin{array}{l}\text { Tomo parte sin } \\
\text { esfuerzo en } \\
\text { cualquier } \\
\text { conversación o } \\
\text { debate y conozco } \\
\text { bien modismos, } \\
\text { frases hechas y } \\
\text { expresiones } \\
\text { coloquiales. Me } \\
\text { expreso con fluidez } \\
\text { y transmito matices } \\
\text { sutiles de sentido } \\
\text { con precisión. Si } \\
\text { tengo un problema, } \\
\text { sorteo la dificultad } \\
\text { con tanta discreción } \\
\text { que los demás } \\
\text { apenas se dan } \\
\text { cuenta. }\end{array}$ & \begin{tabular}{|l} 
Presento \\
descripciones \\
o argumentos \\
de forma clara \\
y fluida y con \\
un estilo que \\
es adecuado al \\
contexto y con \\
una estructura \\
lógica y eficaz \\
que ayuda al \\
oyente a \\
fijarse en las \\
ideas \\
importantes y \\
a recordarlas.
\end{tabular} & \begin{tabular}{|l} 
Soy capaz de \\
escribir textos \\
claros y fluidos en \\
un estilo \\
apropiado. Puedo \\
escribir cartas, \\
informes o \\
artículos \\
complejos que \\
presentan \\
argumentos con \\
una estructura \\
lógica y eficaz que \\
ayuda al oyente a \\
fijarse en las ideas \\
importantes y a \\
recordarlas. \\
Escribo \\
resúmenes y \\
reseñas de obras \\
profesionales o \\
literarias.
\end{tabular} \\
\hline
\end{tabular}

Sobre los grados de correlación y equivalencia del MCE con otras pruebas estandarizadas para los idiomas español, inglés y francés correspondientes a cursos o programas de instrucción, se tienen los siguientes,

Tabla 6. Equivalencias del nivel MCE con varias pruebas para español, inglés y francés

\begin{tabular}{|c|c|c|c|c|c|c|}
\hline Lengua & A1 & $\mathrm{A} 2$ & B1 & B2 & C1 & $\mathrm{C} 2$ \\
\hline Español & \begin{tabular}{|l} 
Diploma de \\
Español \\
Nivel A1) \\
\end{tabular} & \begin{tabular}{|l} 
Diploma de \\
Español (Nivel \\
$\underline{A} 2$ )
\end{tabular} & \begin{tabular}{|l} 
Diploma de \\
Español (Nivel \\
B1. Inicial)
\end{tabular} & \begin{tabular}{|l|} 
Diploma de \\
Español (Nivel \\
B2. \\
Intermedio) \\
\end{tabular} & \begin{tabular}{|l} 
Diploma de \\
Español (Nivel \\
C1)
\end{tabular} & \begin{tabular}{|l} 
Diploma de \\
Español (Nivel \\
C2. Superior) \\
\end{tabular} \\
\hline Inglés & \begin{tabular}{|l} 
ESOL 1-2; \\
Trinity \\
Grades 1,2; \\
Ascentis \\
Anglia ESOL \\
Preliminary \\
Level
\end{tabular} & \begin{tabular}{|l} 
Key English \\
Test (KET); \\
Young \\
Learners; \\
BULATS 1; \\
ESOL 3-4; \\
Trinity Grades \\
3,4; Ascentis \\
Anglia ESOL \\
Elementary \\
Level
\end{tabular} & $\begin{array}{l}\text { Preliminary } \\
\text { English Test } \\
\text { (PET); BEC 1; } \\
\text { BULATS 2; ESOL } \\
\text { 5-6; Trinity } \\
\text { Grades 5,6; } \\
\text { Ascentis Anglia } \\
\text { ESOL } \\
\text { Intermediate } \\
\text { Level }\end{array}$ & \begin{tabular}{|l|} 
First Certificate \\
in English \\
(FCE); BEC 2; \\
BULATS 3; \\
ESOL 7-9; \\
Trinity Grades \\
7,8,9; Ascentis \\
Anglia ESOL \\
Advanced Level
\end{tabular} & \begin{tabular}{|l|} 
Certificate in \\
Advanced \\
English (CAE); \\
BEC 3; BULATS \\
3; ESOL 10-11; \\
Trinity Grades \\
10,11; Ascentis \\
Anglia \\
Proficiency \\
Level
\end{tabular} & \begin{tabular}{|l} 
Certificate of \\
Proficiency in \\
English (CPE); \\
BULATS 5; \\
ESOL 12; \\
Trinity Grades \\
$12 ;$ Ascentis \\
Anglia \\
Masters Level
\end{tabular} \\
\hline
\end{tabular}


Fundamentos para el desarrollo profesional de los profesores de inglés. 2ạ. Edición. Pasto: Editorial Universitaria - Universidad de Nariño. (361 pp.). 2015. ISBN 978-958-8609-98-0.

\begin{tabular}{|c|c|c|c|c|c|c|}
\hline Francés & \begin{tabular}{|l}
$\frac{\text { Diplôme }}{\text { d'Etudes en }}$ \\
Langue \\
Française \\
(DELF A1)
\end{tabular} & $\begin{array}{l}\text { Diplôme } \\
\text { d'Etudes en } \\
\text { Langue } \\
\text { Française } \\
\text { (DELF A2) }\end{array}$ & \begin{tabular}{|l}
$\frac{\text { Diplôme }}{\text { d'Etudes en }}$ \\
Langue \\
Française (DELF \\
B1)
\end{tabular} & \begin{tabular}{|l} 
Diplôme \\
d'Etudes en \\
Langue \\
Française \\
(DELF B2)
\end{tabular} & \begin{tabular}{|l} 
Diplôme \\
Approfondi de \\
Langue \\
Française \\
(DALF C1)
\end{tabular} & \begin{tabular}{|l} 
Diplôme \\
Approfondi de \\
Langue \\
Française \\
(DALF C2)
\end{tabular} \\
\hline
\end{tabular}

\section{CONCLUSIÓN}

No solo la evidencia observable sino el sentido común muestra que todo proceso en marcha o post-facto tiene que ser evaluado de manera sistemática de la mejor manera posible con miras a ajustar las metas, los objetivos, contenidos y metodologías utilizadas ya sea de un sistema o de un programa educativo particular. Las pruebas estandarizadas para la lengua extranjera, como medios válidos y confiables de evaluación diseñados, producidos y observados por instituciones y agencias internacionales con trayectoria investigativa son medios necesarios para conocer el estado de desarrollo de las competencias lingüísticas y comunicativas en inglés de los docentes y estudiantes de un sistema educativo. Determinar si los programas educativos a nivel básico están teniendo éxito es una responsabilidad de las instituciones gubernamentales del caso. Las pruebas de tipo estándar contribuirían para que tanto administradores educativos como docentes tomen cursos de acción sobre aspectos curriculares para mejorar la calidad de la educación en lenguas extranjeras. Cada día que pasa, en un mundo globalizado las pruebas estandarizadas se convierten a nivel mundial en un requisito tanto de instituciones como de gobiernos y estados. Es parte importante de la función gubernamental educativa conocer de la manera más objetiva posible (cuantitativa y cualitativamente) el estado de docentes y estudiantes en cuanto a su desarrollo de estas competencias para brindar la capacitación necesaria a los primeros y la garantía de un mejor sistema de educación en lengua extranjera a los segundos.

El Ministerio de Educación Nacional ha dado pasos importantes en la adecuación y revisión de pruebas en este nuevo milenio para determinar el nivel del inglés en estudiantes y profesores de los diferentes niveles de educación, especialmente con la adopción del Marco Común Europeo de Referencia para las Lenguas como referente internacional. Sin embargo, 
Fundamentos para el desarrollo profesional de los profesores de inglés. 2a adición. Pasto: Editorial Universitaria - Universidad de Nariño. (361 pp.). 2015. ISBN 978-958-8609-98-0.

falta la incorporación o adopción estándar de los instrumentos o pruebas y que además puedan ser accesibles en línea a través de Internet, pues ya existe la tecnología en diferentes centros educativos del país. De esta manera estaremos entrando en la cultura de la evaluación estandarizada válida y confiable para determinar cursos de acción en pro de la calidad del desarrollo de la lengua extranjera para fines del progreso social del país.

\section{BIBLIOGRAFÍA}

Allan, D. (2004). The Oxford Placement Test. Oxford: Oxford University Press.

Brown, G., \& Yule, G. (1983). Teaching the spoken language: an approach based on the analysis of conversational English. Cambridge; New York: Cambridge University Press.

The British Council. (2006). Diagnóstico del nivel de Inglés de los docentes de inglés del municipio de San Juan de Pasto.

Dulay, H. C., Burt, M. K., \& Krashen, S. D. (1982). Language two. New York: Oxford University Press.

Hakuta, K., Butler, Y., \& Daria, W. (2000). How long does it take English learners to attain proficiency?

Hays, W. L. (1973). Statistics for the social sciences (2d ed.). New York: Holt.

Hughes, A. (2003). Testing for language teachers (2nd ed.). Cambridge; New York: Cambridge University Press.

Instituto Cervantes (2002). Marco Común Europeo de Referencia para las Lenguas. Consultado desde:

http://cvc.cervantes.es/ensenanza/biblioteca ele/marco/cvc mer.pdf: mayo 17, 2011.

Madsen, H. S. (1983). Techniques in testing. New York, NY: Oxford University Press.

MINISTERIO DE EDUCACIÓN NACIONAL, Colombia Aprende. Consultado Desde: http://www.colombiaaprende.edu.co/html/micrositios/1752/w3propertyname-3009.html.: enero 25, 2010.

Storey, A. G. (1970). The measurement of classroom learning. Chicago, III.: Science Research Associates.

Thorndike, R. M. (1997). Measurement and evaluation in psychology and education (6th ed.). Upper Saddle River, N.J.: Merrill. 
Fundamentos para el desarrollo profesional de los profesores de inglés. 2a adición. Pasto: Editorial Universitaria - Universidad de Nariño. (361 pp.). 2015. ISBN 978-958-8609-98-0.

Tuckman, B. W. (1999). Conducting educational research (5th ed.). Fort Worth, TX: Harcourt Brace College Publishers.

Underhill, N. (1987). Testing spoken language: a handbook of oral testing techniques. Cambridge Cambridgeshire; New York: Cambridge University Press. 